\title{
Comparative antibody study for antigen detection in urine specimens for diagnosis of blastomycosis using a competitive enzyme-linked immunosorbent assay
}

\author{
Amanda Searle*, Gene Scalarone \\ Idaho State University, Pocatello, USA; ${ }^{*}$ Corresponding Author: hillama2@isu.edu, scalgene@isu.edu
}

Received 26 July 2012; revised 7 September 2012; accepted 26 September 2012

\begin{abstract}
Diagnosis of blastomycosis is often done using a combination of clinical signs and cytologic or histopathologic identification of the organism, Blastomyces dermatitidis, from infected tissues. However, these methods are time consuming, invasive, and still lead to misdiagnosis. A competitive enzyme-linked immunosorbent assay (ELISA) can be used for detection of $B$. dermatitidis antigens, which are present in urine specimens of infected patients. The current study evaluates the use of various antibodies for detection of antigen in dog urine specimens, to provide a better diagnosis of blastomycosis in the future. Our results show that different antibodies against $B$. dermatitidis produce various sensitivities for antigen detection. The most realistic antibodies for immunodiagnostic tests would be antibodies that can be obtained in larger quantities, i.e. vaccination using a yeast lysate in a laboratory setting. We found that these antibodies produce a comparable and reliable result to that of antibodies obtained from an infected patient.
\end{abstract}

Keywords: Blastomycosis; ELISA; Antigen Detection

\section{INTRODUCTION}

Blastomycosis is a fungal infection caused by the thermally dimorphic fungus Blastomyces dermatitidis. $B$. dermatitidis grows in moist, slightly acid areas where rich organic matter is present $[1,2]$. In this environment it grows in a mycelial form and produces microscopic spores known as conidia. B. dermatitidis is common to areas of North American including the Great Lakes, parts of Canada, and states ranging from Arkansas to North Carolina [3-7]. A mammal may inhale the conidia from the environment, particularly if there is a disturbance of the soil. Inside the warm, moist environment of the lung, the conidia will convert into the yeast phase of $B$. dermatitidis [8]. In some patients the immune system will resolve the infection relatively fast, while in others it can progress and spread throughout the body. In these cases blastomycosis can be fatal if not treated.

Diagnosis of blastomycosis is often done by a combination of clinical signs and cytologic or histologic identification of the organism [6,9]. Clinical signs are often slow to appear in patients, not revealing a problem until the disease has spread to additional parts of the body. These signs can include fever, anorexia, coughing, dyspnea, ocular and nasal discharge, loss of body condition, cutaneous lesions, and lameness [8]. Unfortunately these signs are not specific to a $B$. dermatitidis infection, but are common to a variety of diseases ranging from bacterial infections to autoimmune diseases. Cytologic and histologic identification is often done using a sputum sample or tissue sample from a patient. These are then observed microscopically. B. dermatitidis will be seen as a large yeast cell that may have a broad-based bud coming off of it. This however can be easily mistaken as different fungal pathogens including Cryptococcus neoformans or Histoplasma capsulatum. To confirm the culprit of the infection, often an additional test is done by culturing the organism and using a chemiluminescent DNA probe (AccuProbe; GenProbe Inc., San Diego, CA) [10].

Due to the invasiveness, time used for growth and testing, and often misdiagnosis of blastomycosis; there is a continual drive to develop new tests that will provide rapid and reliable results. Antigen testing may be a useful way of diagnosing blastomycosis. Antigen detection has been used to identify histoplasmosis, a fungal disease caused by $H$. capsulatum [11]. Fungal antigens can be found in blood of infected patients as well as in urine 
specimens. These antigens will often decrease with treatment and increase with relapse [12].

A competitive enzyme-linked immunosorbent assay (ELISA), shown in Figure 1, can be used to detect antigen in urine samples. A competitive antigen works by allowing the $B$. dermatitidis antigen in the sample to bind with the primary antibody. Then this mixture is added to a plate coated with additional $B$. dermatitidis antigens. Any primary antibody not bound to urine antigens will bind to the plate antigens. Washing the plate removes any compounds or substance not bound to the plate. Next an anti-primary antibody is added, which also contains an enzyme conjugate. This antibody will bind to the primary antibody, and washing following this step removes any that remain unbound. Next a substrate is added. This substrate binds to the conjugate enzyme, causing a color change that is relative to the amount of secondary antibody bound to the plate. Following this step, absorbance is read to measure the quantitative color change. For the control, no antigen (urine specimen) is added, causing all antibodies to bind to the plate. When interpreting results, specimens with more antigen detected with have a lower absorbance reading.

In addition to a competitive ELISA, some assays involve the use of antibody detection from serum specimens of infected patients [13-15]. As the immune system begins to combat the $B$. dermatitidis infection, cells will produce antibodies that are specific to the yeast antigens. Generally these antibody levels are high enough to be detected by a variety of sensitive and specific assays [13, 14]. With immunocompromised patients, there is commonly a much lower level of antibody specific to $B$. dermatitidis cells that is difficult to detect using this assay. In these cases a different type of assay is required for the diagnosis of blastomycosis, such as the detection of antigen from urine specimens. Therefore the use of a competitive ELISA antigen detection assay may provide reliable results for the immunodiagnosis of blastomycosis

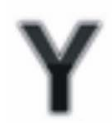

Blastomyces dermatitidis Dog or Rabbit antibody

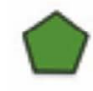

\section{Blastomyces dermatitidis} antigen

Various additional particles in
urine

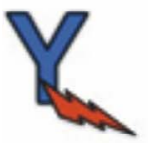

Anti-Dog or Rabbit antibody with enzyme conjugate

Substrate causes color change with conjugate enzyme

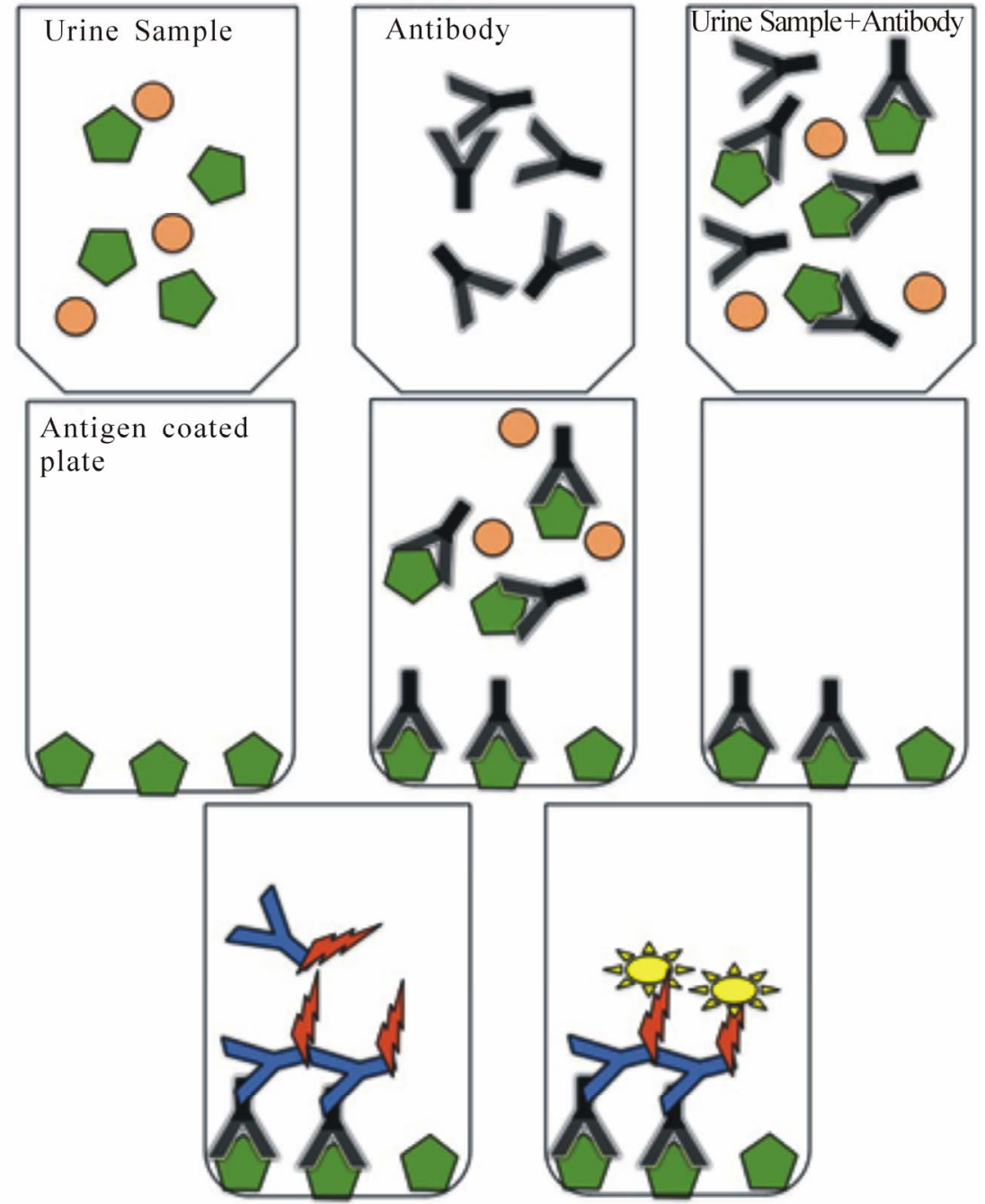

Figure 1. The steps of a competitive ELISA. 
in humans and animals.

Antibodies against $B$. dermatitidis can be obtained from serum of infected patients or immunized individuals. Ideally, serum obtained from an immunized animal would be able to provide large quantities for lab testing. This, however, may not be as reliable at detecting $B$. dermatitidis. This study evaluates the uses of antibodies obtained from infected dog serum specimens as well as antibodies from immunized rabbits in the detection of antigen from dog urine specimens with a competitive ELISA.

\section{MATERIALS AND METHODS}

\subsection{Urine Specimens}

The dog urine specimens were provided by A. M. Legendre (University of Tennessee College of Veterinary Medicine, Knoxville, TN). Urine specimens were from several dogs at various stages of blastomycosis from several locations within endemic areas of North America. These urine samples were taken prior to treatment of the dogs. Each urine specimen is designated with a value for each test run. These specimens vary for each experiment, meaning the Specimen A in Figure 2 is different than Specimen A in Figure 3; due to the quantity available for testing of each specimen.

\subsection{Serum Specimens}

Serum containing antibodies against $B$. dermatitidis were collected previous to this study from New Zealand White rabbits. Rabbits were immunized with a yeast lysate of $B$. dermatitidis. In addition, a number of serum specimens from dogs with blastomycosis were provided by Dr. A. M. Legendre (University of Tennessee College of Veterinary Medicine, Knoxville, TN). Dog serum specimens were from dogs at different stages of blastomycosis, and were all taken prior to antifungal treatment. Serum was used in various dilutions, the most common

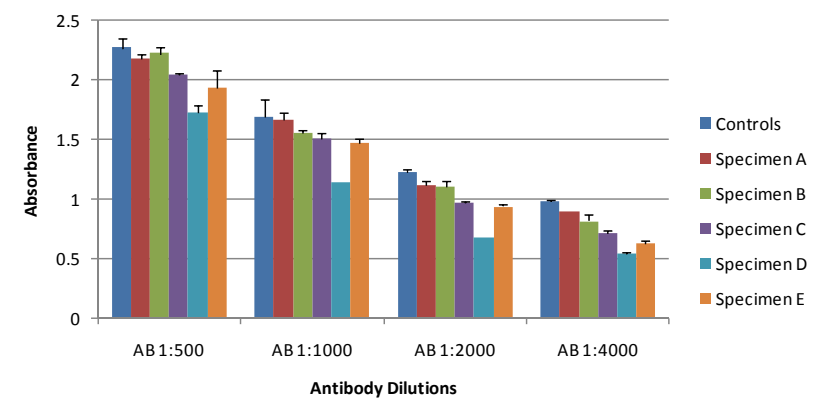

Figure 2. Antibody dilutions of rabbit sera. The control showed no antigen detection; the lower the absorbance values for the specimens the greater antigen detection. All specimens are dog urine samples from dogs at various stages of blastomycosis. Error bars represent standard error.

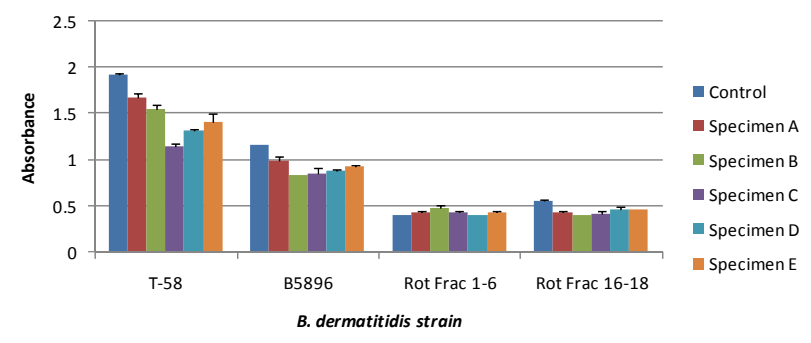

(a)

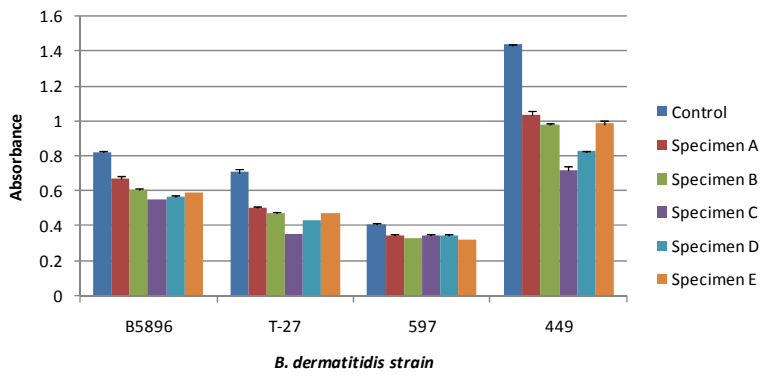

(b)

Figure 3. Various antibodies generated from different strains of $B$. dermatitidis. The control showed no antigen detection; the lower the absorbance values for the specimens the greater antigen detection. All specimens are dog urine samples from dogs at various stages of blastomycosis. Error bars represent standard error. Specimen designation does not correlate to Figure 2, but does correlate for both (a) and (b). Absorbance cannot be compared directly between Figures 3(a) and (b) due to reagent reaction time during the final steps of the competitive ELISA. (a) Detection for 4 different serum specimens. The later two were generated using a rotofor that has been used by differentiate between $B$. dermatitidis antigen based on molecular charge [16]; (b) Detection for four different serum specimens, one of which was shown in Figure 3(a).

being 1:1000; then when added to the urine specimen reached a final dilution of 1:2000. Dilutions were performed using phosphate buffered saline containing $0.15 \%$ Tween 20 (PBS-T).

\subsection{Competitive Enzyme-Linked Immunosorbent Assay}

Serum specimens contain antibody were diluted to various amounts then added to urine specimens. This mixture was allowed to incubate from 30 minutes at $37^{\circ} \mathrm{C}$. For a control and baseline for detection, PBS-T was used in place of urine. Samples were then added in triplicate to a Costar 96-well microplate (Thermo-Fisher Scientific; Waltham, MA); which was coated with $B$. dermatitidis antigen (2000 ng per $\mathrm{ml}$ ) diluted in a carbonate-bicarbonate coating buffer $(\mathrm{pH} 9.6)$ and incubated overnight at $4{ }^{\circ} \mathrm{C}$ in a humid chamber followed by washing three times with phosphate buffered saline containing $0.15 \%$ Tween 20 (PBS-T). Following this addition, the plate was incubated at $37^{\circ} \mathrm{C}$ for 30 minutes. After incubation, wells were washed as above and $100 \mu \mathrm{l}$ of goat 
anti-species (either dog or rabbit) $\operatorname{IgG}(\mathrm{H} \& \mathrm{~L})$ peroxidase conjugate was added to each well and incubated for 30 minutes at $37^{\circ} \mathrm{C}$. The plate was washed again as above and $100 \mu \mathrm{l}$ of peroxidase substrate was added to each well and incubated for approximately 2 minutes at room temperature. The reaction was then stopped by the addition of $2 \mathrm{~N}$ sulfuric acid. The absorbance was read at 450 $\mathrm{nm}$ using a BIO-RAD 2550 EIA reader (Hercules, CA).

\section{RESULTS}

\subsection{Immunized Rabbit Antibodies}

Four different serum specimens were used from rabbits immunized with $B$. dermatitidis yeast lysates. To identify the quantity of serum needed for adequate antigen detection with the competitive ELISA, serum was first tested using a variety of dilutions. Serum specimens were diluted to 1:250, 1:500, 1:1000, and 1:2000. These were then added to dog urine specimens to make the final serum dilution 1:500, 1:1000, 1:2000, and 1:4000. These results are shown in Figure 2.

As the concentration of the antibody was decreased we see lower absorbance values for the control in addition to the urine specimens. This is due to the amount of antibody binding to the antigens on the plate and the antibody still in solution. As the concentration changes, the amount of antibody that binds to the plate will change based on equilibrium. Based on these results, additional tests were done using a final serum dilution of 1:2000. This showed greater antigen detection than other dilutions used.

Additional immunized rabbit sera were tested to identify an optimal serum for antigen detection, thus allowing for greater sensitivity of the competitive ELISA. Serum was used from rabbits immunized with a variety of yeast lysates made using different $B$. dermatitidis strains. Comparative analyses of these different serum specimens are shown in Figure 3.

Figure 3 was done in two different ELISA plates at different times. Because of this, the absorbance readings from one plate cannot be directly compared to that of the other plate, shown in Figures 3(a) and (b) respectively. In both tests serum specimen B5896 was used. These do not show the same absorbance values to the slightly variable incubation of the reagents used in the test that stops direct comparison of absorbance values. Based on these results serum specimens generated from strain 449 and T-58 exhibited the greatest antigen detection. There was no difference in the sensitivity of these antibody specimens; however the specimen generated from strain 449 showed a greater difference between the control and the urine specimens. Therefore, it was used for further comparative analyses with antibodies from infected dog serum.

\subsection{Infected Dog Antibodies}

Antibodies from dogs infected with blastomycosis were tested using the competitive ELISA for antigen detection. Serum contain these antibodies was diluted to 1:1000, making a final dilution of 1:2000 when urine specimens were added. Different dog serum specimens were tested to compare the relative antigen detection and identify the serum that would provide a sensitive result. These results are shown in Figure 4. Based on these results serum specimens 106 and 147 were optimal for antigen detection. Because serum specimen 147 had a relative higher absorbance it was used for further comparisons with immunized rabbit sera.

\subsection{Comparative Analysis of Immunized Serum vs. Infected Serum}

Infected dog serum specimen 147 was compared to immunized rabbit serum generated from $B$. dermatitidis strain 449. Using the competitive ELISA, both serum specimens were used to detect antigen in 13 different infected dog urine samples. These results are shown in Figure 5. The dog serum was capable to detecting anti gen in $76.9 \%$ of the specimens, while the rabbit serum was able to detect antigen in $92.3 \%$ of the specimens.

\section{DISCUSSION}

There is a need for a more improved assay for the diagnosis of blastomycosis. One possible approach is by antigen detection using the competitive ELISA. Our results showed that the use of different antibodies obtained from either immunization against $B$. dermatitidis or infection generate various antigen detection sensitivities. Based on the use of a variety of different antibodies from serum specimens ranging from rabbits immunized with

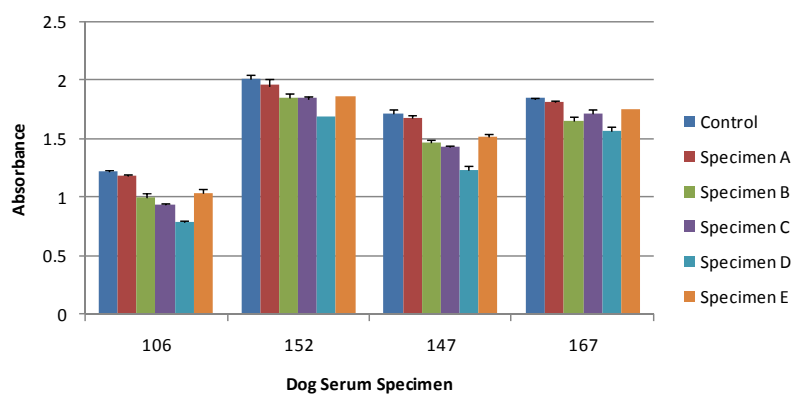

Figure 4. Various antibodies from serum of different infected dogs. The control showed no antigen detection; the lower the absorbance values for the specimens the greater antigen detection. All specimens are dog urine samples from dogs at various stages of blastomycosis. Error bars represent standard error. Specimen designation does not necessarily correlate to Figure 2 or 3. Absorbance cannot be compared between figures due to reagent reaction time during the final steps of the competitive ELISA. 


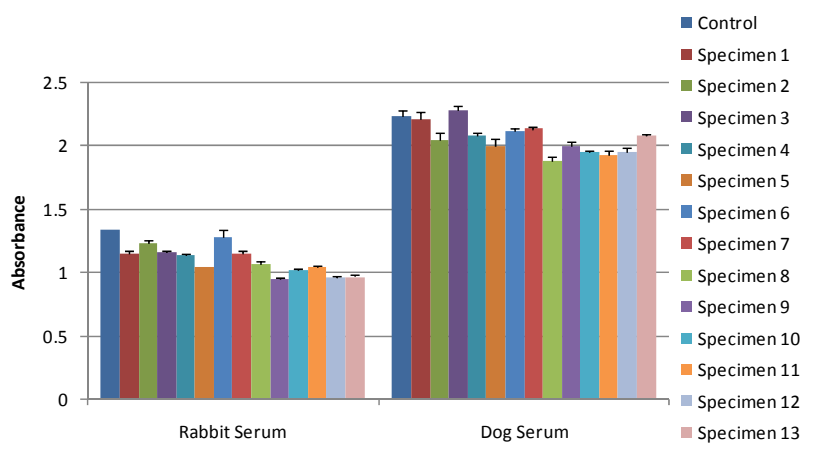

Figure 5. Serum specimen 147 represents infected dog serum while serum specimen 449 represents immunized rabbit serum. The control showed no antigen detection; the lower the absorbance values for the specimens the greater antigen detection. All urine specimens are dog urine samples from dogs at various stages of blastomycosis. Error bars represent standard error. Absorbance cannot be compared between figures due to reagent reaction time during the final steps of the competitive ELISA.

B. dermatitidis yeast cells or from dogs with blastomycosis, we were able to detect antigen at various levels from dog urine samples. The use of different antibodies produces a different percentage of specimens that are identified as positive for $B$. dermatitidis antigens, as seen in Figures 2-5.

Our results show that the use of immunized rabbit serum for the detection of $B$. dermatitidis antigens from dog urine samples produced antigen detection that was comparable to that of infected dog serum. By using the optimal immunized rabbit serum we were able to detect antigen in $92.3 \%$ of the specimens; while using the optimal infected dog serum we detected antigen in $76.9 \%$ of the urine specimens. Based on these results the immunized rabbit sera had a greater sensitivity for antigen detection than that of the infected dog serum. Thus, future antigen detection tests that utilize rabbit serum, such as our rabbit serum specimen 449 , should produce a highly sensitive assay for diagnosing blastomycosis. Studies are continuing in an effort to further optimize this antigen detection assay.

\section{ACKNOWLEDGEMENTS}

This research was supported by the University Research Committee Grant No. S11-6U and the Department of Biological Sciences at Idaho State University, Pocatello, Idaho.

\section{REFERENCES}

[1] Baumgardner, C.D.J. and Paretsky, D.P. (1999) The in vitro isolation of Blastomyces dermatitidis from a woodpile in north central Wisconsin, USA. Medical Mycology, 37, 163-168.

[2] Klein, B.S., Vergeront, J.M., Weeks, R.J., Kumar, U.N., Mathai, G., Varkey, B., et al. (1986) Isolation of B. dermatitidis in soil associated with a large outbreak of blas- tomycosis in Wisconsin. The New England Journal of Medicine, 31, 4529-4534.

[3] Center for Disease Control (1996) Blastomycosis-Wisconsin, 1986-1995. Morbidity and Mortality Weekly Report, 45, 601-603.

[4] Center for Disease Control (1999) Blastomycosis acquired occupationally during prairie dog relocation-Colorado, 1998. Morbidity and Mortality Weekly Report, 48, 98-100.

[5] Center for Disease Control (2012) Geographic distribution of endemic fungal infections among older persons, United Sates. Emerging Infectious Diseases, 18, 2. http://wwwnc.cdc.gov/eid/article/18/2/11-1537_article.htm

[6] McCullough, M.J., DiSalvo, A.F., Clemons, K.V., et al. (2000) Molecular epidemiology of Blastomyces dermatitidis. Clinical Infectious Diseases, 30, 328-335. doi: $10.1086 / 313649$

[7] Reed, K.D., Meece, J.K., Archer, J.R. and Peterson, A.T. (2008) Ecological niche modeling of Blastomyces dermatitidis in Wisconsin. PLos One, 3, e2034. doi:10.1371/journal.pone.0002034

[8] Di Salvo, A.F. (1998) Chapter 18 in Topley and Wilson's microbiology and microbial infections. Arnold Publishers, London.

[9] Spector, D., Legendre, A.M., Wheat, J., Bemis, D., Rohrbach, B., Taboada, J. and Durkin, M. (2008) Antigen and antibody testing for the diagnosis of blastomycosis in dogs. Journal of Veterinary Internal Medicine, 22, 839843. doi:10.1111/j.1939-1676.2008.0107.x

[10] Saccente, M. and Woods, G.L. (2010) Clinical and laboratory update on blastomycosis. Clinical Microbiology Review, 23, 367-381. doi:10.1128/CMR.00056-09

[11] Wheat, L.J. (2006) Improvement in diagnosis of histoplasmosis. Expert Opinion on Biological Therapy, 6, 1207-1221. doi:10.1517/14712598.6.11.1207

[12] Mongkolrattanothai, K., Peev, M., Wheat, L.J. and Marcinak, J. (2006) Urine antigen detection of blastomycosis in pediatric patients. The Pediatric Infectious Disease Journal, 25, 1076-1078. doi:10.1097/01.inf.0000241144.89426.2a

[13] Chester, E.M., Axtell, R.C. and Scalarone, G.M. (2003) Blastomyces dermatitidis lysate antigens: Antibody detection in serial serum specimens from dogs with blastomycosis. Mycopathologia, 156, 289-294. doi:10.1023/B:MYCO.0000003575.04692.2f

[14] Sestero, C.M. and Scalarone, G.M. (2006) Detection of $\operatorname{IgG}$ and $\operatorname{IgM}$ in sera from canines with blastomycosis using eight $B$. dermatitidis yeast phase lysate antigens. Mycopathologia, 162, 33-37. doi:10.1007/s11046-006-0028-7

[15] Shurley, J.F., Legendre, A.M. and Scalarone, G.M. (2005) Blastomyces dermatitidis antigen detection in urine specimens from dogs with blastomycosis using a competitive binding inhibition ELISA. Mycopathologia, 160, 137-142. doi:10.1007/s11046-005-3153-9

[16] Hayden, J. (2011) Comparative antibody detection studies using isoelectrically focused protein fractions from Blastomyces dermatitidis. M.S. Thesis, Idaho State University, Pocatello. 